\title{
EFEITO DA GOMA XANTANA E DA BENTONITA NO DESEMPENHO DE UM FLUIDO DE PERFURAÇÃO BASE MICROEMULSÃO
}

R.P.F. SOUSA ${ }^{1}$, F.D.S. CURBELO ${ }^{2}$, A.I.C. GARNICA ${ }^{3}$, E.A. ARAÚJO ${ }^{4}$, J.C.O. FREITAS, ${ }^{5}$ G.S. BRAGA ${ }^{6}$

Universidade Federal da Paraíba ${ }^{1,2,3}$, Universidade Federal do Rio Grande do Norte ${ }^{4,5}$, Instituto Federal de Educação, Ciência e Tecnologia do Maranhão ${ }^{6}$ roxana.fp@gmail.com ${ }^{1}$

Submetido 30/05/2018 - Aceito 16/03/2020

DOI: $10.15628 /$ holos.2020.7358

\section{RESUMO}

Os fluidos de perfuração microemulsionados são uma nova tecnologia que objetiva substituir os fluidos base óleo e base água, por possuir alta lubricidade, estabilidade, baixa toxicidade, baixo custo e menor perda de fluido. Assim, este trabalho teve como objetivo o estudo da ação viscosificante da goma xantana e da bentonita em um fluido de perfuração microemulsionado, composto por uma solução aquosa de glicerina, um óleo vegetal e um tensoativo não iônico, através de testes reológicos. A partir dos resultados, concluiu-se que a goma xantana apresenta maior ação viscosificante, uma vez que o fluido aditivado com uma menor quantidade deste biopolímero apresentou maior viscosidade aparente e plástica, força gel e limite de escoamento. Os dois viscosificantes atribuíram pseudoplasticidade aos fluidos e o modelo reológico que melhor se ajustou aos comportamentos apresentados foi o modelo de Herschel-Bulkley.

PALAVRAS-CHAVE: fluido de perfuração, goma xantana, bentonita, microemulsão

\section{EFFECTS OF XANTHAN GUM AND BENTONITE ON THE PERFORMANCE OF A MICROEMULSION-BASED DRILLING FLUID}

\begin{abstract}
Microemulsion-based drilling fluids are a new technology that aims to substitute water and oil-based drilling fluid, for featuring high lubricity, stability, low toxicity, low cost and less fluid loss. This paper studies the viscosifying action of xanthan gum and bentonite in a microemulsionbased drilling fluid, composed by an aqueous solution of glycerin, vegetable oil and a nonionic surfactant, through rheological tests. From the results, it could be concluded
\end{abstract}

that the xanthan gum has a higher viscosifying action, since the drilling fluid added with a smaller amount of this biopolymer presented a higher apparent and plastic viscosity, gel strength and shear limit. The two viscosifiers attributed pseudoplasticity to the drilling fluids and the rheological model that better adjusted to the presented behaviors was the Herschel-Bulkley model.

KEYWORDS: drilling fluid, xanthan gum, bentonite, microemulsion. 


\section{INTRODUÇÃO}

Os fluidos de perfuração sintéticos de base orgânica surgiram como uma alternativa às limitações dos fluidos de perfuração base água e para atender as restrições impostas aos fluidos de perfuração base óleo convencionais. Estes fluidos apresentam vantagens ecológicas, como biodegradabilidade e baixa toxicidade. Assim, novas bases sintéticas vêm sendo propostas para formulação de fluidos cada vez menos agressivos ao meio ambiente e que atendam as aplicações das bases aquosas e oleosas convencionais. Como exemplo, têm-se os fluidos de perfuração constituídos de glicerina (Corrêa et al., 2017; Cruz et al., 2015), óleos vegetais (Silva, 2003) e microemulsão contendo glicerina e óleo vegetal (Curbelo et al., 2016).

Os fluidos de perfuração são fluidos viscosos, porém, essa viscosidade deve ser controlada e adequada as necessidades do poço, visto que em algumas situações, ela irá trazer vantagens, enquanto em outras, não. Assim, é importante conhecer o comportamento de fluxo deste fluido e prever o seu comportamento diante das diferentes condições de cisalhamento que serão impostas ao longo da perfuração.

O estudo da deformação e o escoamento de um fluido quando submetido (ou não) a uma tensão externa é chamado de reologia. As relações entre a tensão de cisalhamento aplicada ao fluido e a sua deformação resultante descrevem o comportamento reológico do fluido (Cremasco, 2012).

O fluido de perfuração circula pelo poço e pelo sistema de perfuração de forma a viabilizar uma perfuração rápida, eficiente e segura. Ele deve manter suas propriedades reológicas, executar suas funções e não causar danos à formação, mesmo estando em contato direto com estas rochas.

As microemulsões podem ser definidas como sistemas de alta agregação nas quais água e óleo são homogeneamente misturados pela adição dos tensoativos (Mittal, 1979). A formação da microemulsão envolve, normalmente, a combinação de três a quatro componentes, tais como tensoativo, fase aquosa, fase oleosa e, quando necessário, o cotensoativo (Daltin, 2011). Dentre as vantagens que as microemulsões agregam aos fluidos de perfuração, estão a estabilidade, resistência à corrosão, bons coeficientes de lubricidade e força gel (Hayes et al., 1977; Davies et al., 1997).

Como as propriedades do fluido mudam durante a perfuração, devido à incorporação de fluidos da formação e de sólidos perfurados, o fluido de perfuração deve ser aditivado de modo a alterar suas propriedades reológicas de acordo com as necessidades de cada aplicação. Os aditivos mais utilizados para viscosificar os fluidos são os polímeros e as argilas (Souza et al., 2017; Oliveira et al., 2015; Choo \& Bai, 2015; Nascimento, et al., 2010; Borges \& Vendruscolo, 2009).

A goma xantana é um biopolímero natural, não iônico, produzido por bactérias fitopatogênicas do gênero Xanthomonas sendo Xanthomonas campestres a espécie mais utilizada (Hamed \& Belhadri, 2009; Assis, 2014). As conformações estruturais da molécula são de hélice simples, dupla ou tripla, que interage com outras moléculas de polímero para formar uma rede complexa (Hamed \& Belhadri, 2009). Este biopolímero é utilizado nos fluidos de perfuração como um agente de controle reológico em sistemas aquosos e como estabilizante para emulsões e 
suspensões (Hamed \& Belhadri, 2009), devido as suas distintas propriedades reológicas, tais como alto grau de pseudoplasticidade e elevada viscosidade, mesmo a baixas concentrações, além de possuir vantagens como espessante, estabilizante, gelificante, agente de suspensão e floculação (Araújo et al., 2005; Melo et al., 2013).

A bentonita é adicionada aos fluidos de perfuração não só para controle da viscosidade, como também para auxiliar o transporte dos cascalhos do fundo do poço para a superfície e para controle de filtrado (Kelessidis et al., 2006). Ross \& Shannon (1926) definem bentonita como uma argila constituída essencialmente por um argilomineral montmorilonítico (esmectítico). São materiais naturais, formadas por silicatos hidratados de alumínio, ferro e magnésio que possuem como características principais o alto poder de inchamento, alta área de superfície, capacidade de troca catiônica e tixotropia (Santos, 1989; Melo et al., 2013).

$\mathrm{Na}$ intenção de estudar os efeitos do viscosificante em uma nova base de fluido de perfuração microemulsionada, composta por água, glicerina, óleo vegetal e tensoativo não iônico, foram feitas comparações entre formulações com a goma xantana e a bentonita, em diferentes concentrações, através de testes reológicos, para determinar o melhor viscosificante para o sistema. As composições dos fluidos de perfuração microemulsionados são inovações e estão protegidas pelas patentes de números BR1020160190487 e BR1020180163779, registradas no INPI (Instituto Nacional da Propriedade Industrial).

\section{METODOLOGIA}

A microemulsão utilizada como base para o fluido de perfuração foi composta por $70 \%$ de uma solução aquosa de glicerina P.A. (fase aquosa), 25 \% de tensoativo não iônico e 5 \% de óleo vegetal (fase oleosa).

\subsection{Preparação das soluções de goma xantana}

A fim de analisar a hidratação da goma xantana em uma solução aquosa de glicerina, foram feitos ensaios reológicos de soluções com diferentes concentrações de goma. Os ensaios foram realizados nos intervalos de tempo de $0,24,48$ e 72h após a preparação das soluções de goma, no intuito de verificar a influência do tempo de hidratação desse biopolímero na reologia da solução. Para isso, foram preparadas soluções de 0,82 \%, 1,22 \% e 1,63 \%, em massa, de goma xantana em uma solução aquosa de glicerina. As soluções foram preparadas através da dissolução da goma na fase aquosa através de 30 min de agitação mecânica.

De posse da melhor concentração e do melhor tempo de hidratação da goma, extraídos da análise acima, o fluido de perfuração com goma xantana foi preparado com adição desta goma à fase aquosa da microemulsão, sob agitação constante por $30 \mathrm{~min}$. Em seguida, o sistema foi deixado em repouso para melhor hidratação. Após esse período, foram adicionados ao sistema $\mathrm{NaCl}$ e uma mistura do tensoativo com o óleo vegetal, seguidos da adição dos demais aditivos, redutor de filtrado e adensante, conforme Tabela 1. 
Tabela 1: Formulação base dos fluidos de perfuração estudados.

\begin{tabular}{cc}
\hline Aditivo & Concentração (g/350 $\mathbf{~ L ~ d e ~ m i c r o e m u l s a ̃ o ) ~}$ \\
\hline $\mathrm{NaCl}$ & 17,5 \\
\hline Redutor de filtrado & 3,01 \\
\hline Adensante & 84 \\
\hline
\end{tabular}

\subsection{Preparação das soluções de bentonita}

Da mesma maneira, para analisar o inchamento da bentonita sódica em uma solução aquosa de glicerina, foram feitas soluções aquosas de glicerina com concentrações mássicas de 1,5 $\%, 2,1 \%$ e $2,9 \%$ de bentonita, através da adição da argila à fase aquosa, seguido de 10 min de agitação.

O fluido de perfuração com bentonita foi preparado através da adição da bentonita à água, sob agitação mecânica por $10 \mathrm{~min}$. Em seguida, o sistema foi deixado em repouso por $24 \mathrm{~h}$ para hidratação da argila. Após esse tempo, seguiu-se com adição da glicerina, do tensoativo, óleo vegetal, $\mathrm{NaCl}$, redutor de filtrado e adensante, nas mesmas quantidades utilizadas para o fluido de perfuração preparado com goma xantana, apresentadas na Tabela 1.

\subsection{Análises reológicas}

As massas específicas dos fluidos foram determinadas através de uma balança de lama Fann Modelo 140.

Para análise do comportamento reológico das soluções viscosas e dos fluidos de perfuração, baseado na Norma API 13B (2012), foram efetuadas medidas de viscosidade no viscosímetro Fann, $35 \mathrm{~A}$, utilizado para medir viscosidade aparente (VA) e plástica (VP), limite de escoamento (LE), medidas de gel inicial (GO) e gel final (GF). As leituras foram efetuadas para as seguintes velocidades rotacionais, 600, 300, 200, 100, 6 e $3 \mathrm{rpm}$, permanecendo o fluido $1 \mathrm{~min}$ em cada velocidade para, em seguida, ser feita a leitura da deflexão. Além disso, a partir dessas leituras, foi possível determinar o modelo reológico que melhor se ajusta ao comportamento dos fluidos de perfuração.

O modelo reológico que melhor se adequou aos comportamentos apresentados pelos fluidos foi o modelo de Herschel-Bulkley, que é um modelo de três parâmetros, conforme Equação 1 , sendo eles a tensão de cisalhamento inicial (国), que permite prever a força mínima requerida para iniciar o fluxo; o parâmetro de consistência $(k)$, que mede a resistência do fluido ao escoamento; e o índice de comportamento (n), que mede o afastamento do comportamento do fluido do modelo newtoniano.

$\tau=\tau_{0}+k(\gamma)^{n}$

Para determinar os parâmetros deste modelo, inicialmente estimou-se o valor do por extrapolação através do gráfico de tensão cisalhante (囫) versus taxa de deformação (囫) em

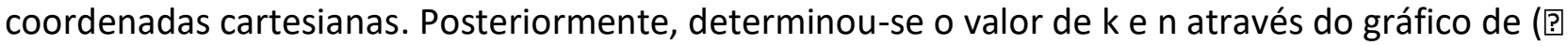
- 国) versus 国, em coordenadas logarítmicas (Machado, 2002). 


\section{RESULTADOS E DISCUSSÃO}

\subsection{Soluções de goma xantana}

A partir da análise da viscosidade plástica e da viscosidade aparente com o tempo de hidratação para as diferentes concentrações de goma xantana na solução aquosa de glicerina, apresentados na Figura 1, pôde-se perceber que, mesmo em baixas concentrações, a goma foi capaz de tornar a solução bastante viscosa. Além disso, é possível perceber que as viscosidades aumentam com a concentração do biopolímero, sendo 1,63 \% a concentração de goma que apresentou maior viscosidade. Segundo García-Ochoa et al. (2000), este comportamento é devido ao aumento da interação molecular, que proporciona um aumento na dimensão efetiva da macromolécula e no peso molecular.

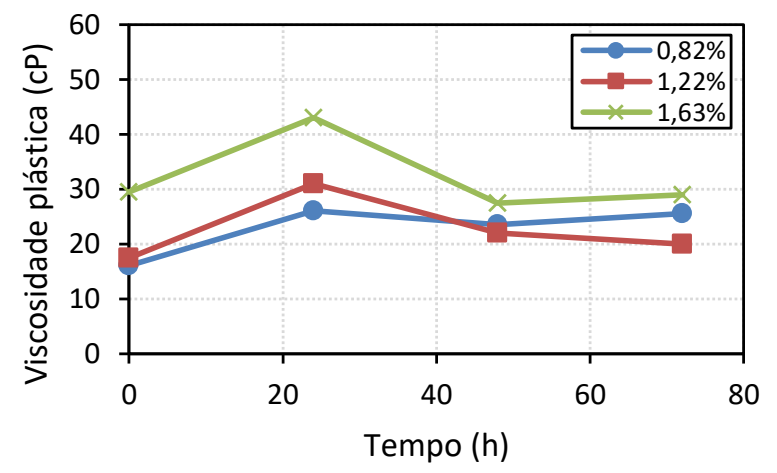

a)

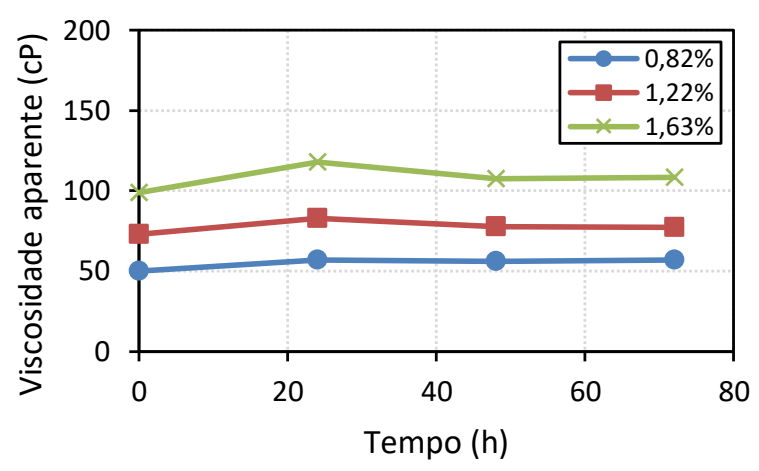

b)

Figura 1: Variação da viscosidade com o tempo de hidratação para as 3 concentrações de goma xantana -

a) Viscosidade plástica; b) Viscosidade aparente.

Ainda de acordo com a Figura 1, foi possível perceber que a viscosidade varia com o tempo de hidratação para todas as concentrações. Até $24 \mathrm{~h}$ de solubilização, ocorre um aumento nas viscosidades. As viscosidades reduzem no intervalo de tempo de 24 a $48 \mathrm{~h}$ e depois tornam-se praticamente constantes. No estado sólido, a goma xantana apresenta cadeias entrelaça das e os movimentos brownianos são limitados. Já em solução, ocorre uma expansão das cadeias, aumentando a liberdade para movimentos translacionais, que são regidos pelas diferentes conformações da molécula (Young, 1981). Assim, pelo comportamento apresentado na Figura 1 , as moléculas do polímero se expandem e se rearranjam nas primeiras $48 \mathrm{~h}$, e atingem uma conformação mais estável para este meio nas horas seguintes, mantendo as viscosidades constantes.

Com isso, para preparação do fluido de perfuração com goma xantana foi escolhido uma concentração de $1,5 \%$ da goma, com tempo de hidratação de $72 \mathrm{~h}$, por apresentar alta viscosidade e estabilidade desse parâmetro com o tempo. 


\subsection{Soluções de bentonita}

O estudo do inchamento de bentonita na solução aquosa de glicerina e água apontou que não há interação entre a argila e a glicerina, uma vez que o inchamento ocorreu apenas na metade do volume da solução para todas as concentrações da bentonita. Este argilomineral é formado por duas folhas de silicato tetraédricas, com uma folha central octaédrica, unidas entre si por oxigênios comuns às folhas (Santos, 1989). O empilhamento dessas placas ocorre por forças polares relativamente fracas e por forças de Van De Waals. Os espaços entre as placas são denominados de espaços interlamelares, nos quais estão cátions trocáveis, como Na+, $\mathrm{Ca} 2+$, Li+ (Silva \& Ferreira, 2008). A argila utilizada no experimento foi uma bentonita sódica, composta por cátions trocáveis $\mathrm{Na}+$ e que, quando expostas à água, as moléculas de água são adsorvidas na superfície das camadas de sílica, provocando o comportamento chamado de inchamento interlamelar que é controlado pelo cátion associado à estrutura (Amorim, 2003), que no caso, é o cátion Na+.

Então, concluiu-se que a glicerina não consegue adsorver nos espaços interlamelares de bentonitas sódicas por não apresentar afinidade química pelo cátion de sódio. Para preparação do fluido, optou-se por inchar 2,14 \% de bentonita, em massa da microemulsão, apenas na água da fase aquosa e deixar em repouso por $24 \mathrm{~h}$. Após esse período, a argila havia inchado em todo o volume de água, então foi possível prosseguir com a adição da glicerina e dos outros componentes do fluido, conforme apresentado na metodologia. O inchamento completo da bentonita no meio é importante na preparação de fluidos de perfuração, para que a base do fluido consiga sustentar os aditivos sólidos adicionados e para manter o fluido estável durante a perfuração, de maneira que ele possa executar suas funções.

\subsection{Fluidos de perfuração}

Foram obtidos dois fluidos de perfuração, denominados FGX e FBT, relativos à adição de goma xantana e bentonita, respectivamente. Os fluidos de perfuração FGX e FBT apresentaram massa específica de $1,12 \mathrm{~g} / \mathrm{cm}^{3}$ e $1,15 \mathrm{~g} / \mathrm{cm}^{3}$, respectivamente, não apresentando diferenças significativas. Os valores ideais desses parâmetros dependem da zona do poço de petróleo a ser perfurada.

\subsubsection{Curvas de consistência}

A partir dos ensaios reológicos dos fluidos de perfuração desenvolvidos, pôde-se construir suas curvas de consistência, apresentadas na Figura 2. De acordo com esta figura, todos os fluidos apresentaram comportamentos de fluidos não-newtonianos, não lineares e com tensão de cisalhamento inicial. O comportamento do FBT tem uma não linearidade ma is suave, e ambos os fluidos apresentaram uma tensão de cisalhamento inicial próxima do zero. 


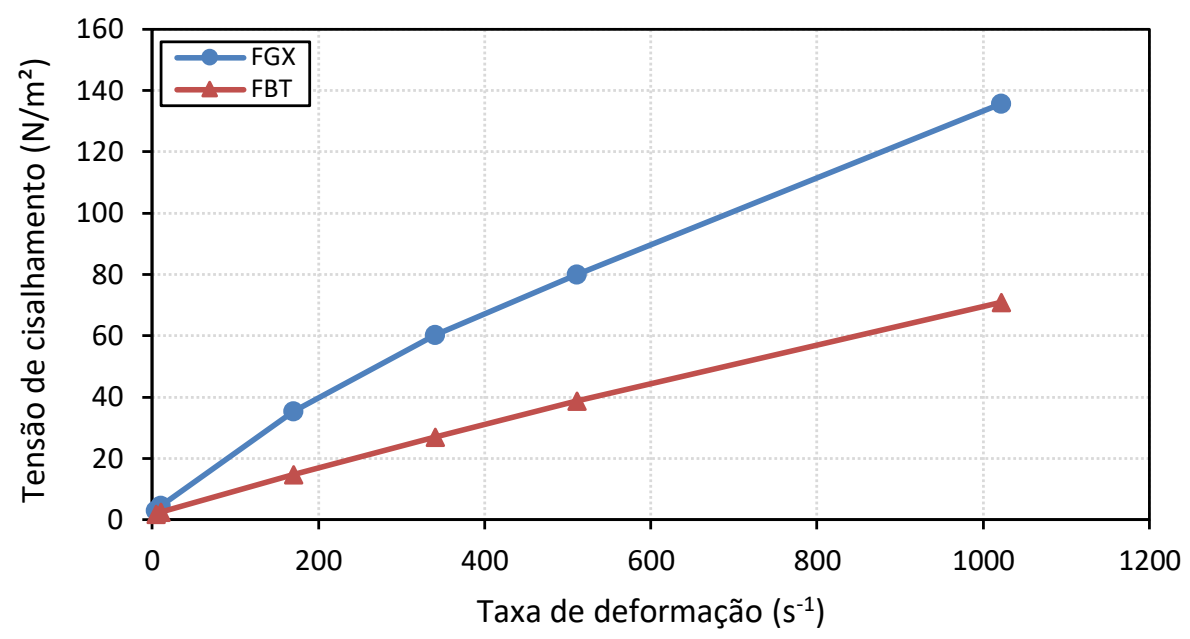

Figura 2: Curvas de consistência para os fluidos FGX e FBT.

\subsubsection{Ajustes do modelo de Herschel-Bulkley}

Conforme visto na Figura 2, os dois fluidos desenvolvidos são não-newtonianos, não lineares e apresentam uma tensão de cisalhamento inicial. Assim, o modelo reológico, que melhor se ajustou aos comportamentos apresentados, foi o modelo de Herschel-Bulkley, que é um modelo de três parâmetros, classificando-os como fluidos de potência com limite de escoamento inicial. Os parâmetros deste modelo, calculados para os fluidos FGX e FBT, estão apresentados na Tabela 2.

Tabela 2: Parâmetros reológicos do modelo de Herschel-Bulkley para os fluidos FGX e FBT.

\begin{tabular}{cccc}
\hline Fluido & $\boldsymbol{\tau}_{\mathbf{0}}\left(\mathbf{l b f} / \mathbf{1 0 0 \mathbf { f t } ^ { \mathbf { 2 } } )}\right.$ & $\mathbf{n}$ & $\mathrm{K}\left(\mathbf{P a} \cdot \mathbf{s}^{\mathbf{n}}\right)$ \\
\hline FGX & 1 & 0,7923 & 0,5713 \\
\hline FBT & 1 & 0,8316 & 0,2087 \\
\hline
\end{tabular}

Através da análise da Tabela 2, percebe-se que o fluido FGX apresentou maior índice de consistência (k), indicando que oferece maior resistência ao escoamento, em relação ao fluido FBT, e apresentou índice de comportamento (n) menor, o que faz com que esse fluido tenha um comportamento mais afastado do modelo newtoniano $(n=1)$ que o fluido FBT. Além disso, observou-se que os dois fluidos apresentaram a mesma tensão de cisalhamento inicial.

Para avaliar a aproximação do modelo de Herschel-Bulkley dos comportamentos apresentados pelos fluidos, foram feitas comparações entre a tensão de cisalhamento experimental e a calculada pelo modelo de Herschel-Bulkley, conforme Figura 3. Estes resultados foram estudados através de análise estatística, apresentada na Tabela 3, usando a ferramenta ANOVA, disponível no programa Excel, com $95 \%$ de confiança. Analisando a figura, percebe-se uma linearidade das curvas e analisando a tabela, pôde-se concluir que as aproximações do modelo 
para os dois fluidos foram estatisticamente significativas, com valores-p inferiores aos níveis de significância, $\alpha(0,05)$, e pares de limites, superiores e inferiores, com o mesmo sinal. Além disso, o modelo melhor se ajustou ao fluido FGX, apresentando maior $\mathrm{R}^{2}$.

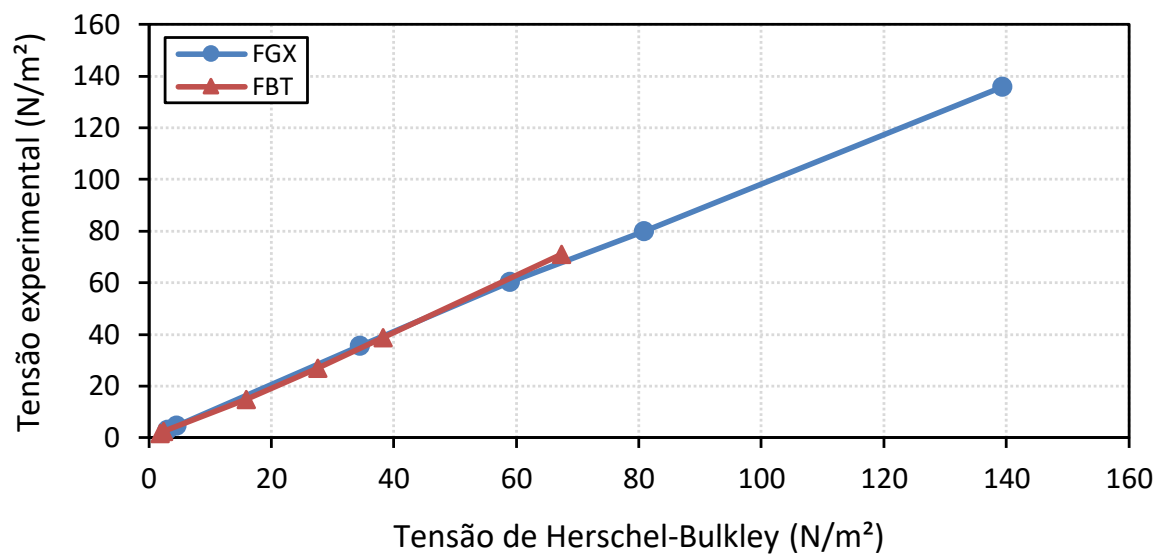

Figura 3: Comparação das tensões do modelo de Herschel-Bulkley e das tensões experimentais para os fluidos FGX e FBT.

Tabela 3: Análise estatística do modelo Herschel-Bulkley para os fluidos FGX e FBT.

\begin{tabular}{|c|c|c|c|c|}
\hline \multirow{2}{*}{ Fluido } & \multirow{2}{*}{$\mathbf{R}^{2}$} & \multirow{2}{*}{ Erro padrão } & valor-p & \multirow{2}{*}{ Limites (sup. e inf.) } \\
\hline & & & $C_{1}$ & \\
\hline FGX & 0,9997 & 1,3916 & $6,478 E-10$ & Significativo \\
\hline FBT & 0,9990 & 1,2483 & 1,179E-08 & Significativo \\
\hline
\end{tabular}

\subsubsection{Pseudoplasticidade}

Para análise da pseudoplasticidade dos fluidos, construiu-se, inicialmente, um gráfico, em escala logarítmica, da viscosidade aparente por taxa de deformação, Figura 4. Através da inspeção desta figura, é possível perceber que os dois fluidos apresentaram comportamento aproximadamente linear e decrescente. A diminuição da viscosidade aparente à medida que a taxa de cisalhamento aumenta é conhecida como afinamento por cisalhamento e normalmente é uma propriedade desejável, porque a viscosidade aparente será relativamente baixa em altas taxas de cisalhamento, que prevalecem no tubo de perfuração, reduzindo assim as pressões de bombeamento e, relativamente alta em baixas taxas de cisalhamento, que prevalecem no espaço anular, aumentando assim, a capacidade de carreamento dos cascalhos. 


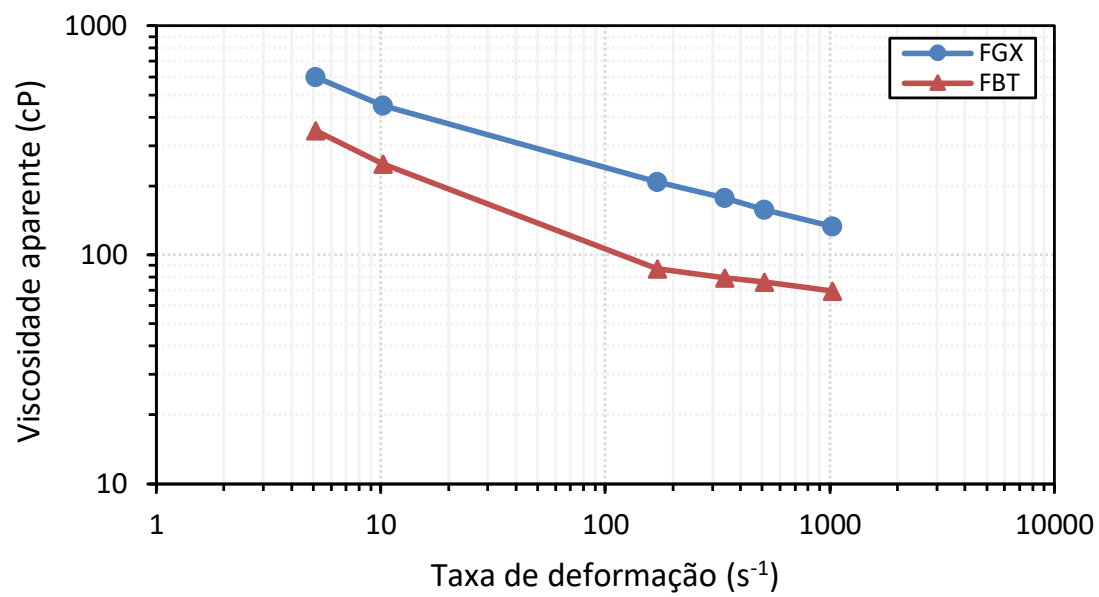

Figura 4: viscosidade aparente versus taxa de deformação, em escala logarítmica, para os fluidos FGX e FBT.

Em escala logarítmica, a curva da viscosidade aparente por taxa de deformação, para fluidos pseudoplásticos, deve apresentar uma reta decrescente. Para analisar a linearidade das curvas de viscosidade, foram feitas regressões lineares através da ferramenta ANOVA, do programa Excel, com $95 \%$ de confiança, resumidas na Tabela 4.

Tabela 4: Análise estatística das regressões lineares das viscosidades dos fluidos FGX e FBT.

\begin{tabular}{cccccc}
\hline \multirow{2}{*}{ Fluido } & $\mathbf{R}^{\mathbf{2}}$ & Erro & \multicolumn{2}{c}{ valor-p } & \multirow{2}{*}{ Limites (sup. $\mathbf{e}$ inf.) } \\
\cline { 5 - 6 } & & padrão & $\mathbf{C}_{\mathbf{0}}$ & $\mathbf{C}_{\mathbf{1}}$ & \\
\hline FGX & 0,9969 & 0,0166 & $6,987 \mathrm{E}-09$ & $3,634 \mathrm{E}-06$ & Significativo \\
\hline FBT & 0,9703 & 0,0588 & $1,517 \mathrm{E}-06$ & $3,352 \mathrm{E}-04$ & Significativo \\
\hline
\end{tabular}

A partir da análise da Tabela 4, é possível perceber que o fluido FGX é mais pseudoplástico que o FBT. Este comportamento está em concordância com o resultado da Tabela 2, em que se concluiu que o fluido FGX apresentou maior afastamento do comportamento newtoniano, sendo a pseudoplasticidade $(0<n<1)$ uma característica de fluidos não-newtonianos. De acordo com KiWon e colaboradores (2006), este comportamento é decorrente do uso da goma xantana no fluido, no qual a viscosidade diminui com o aumento da taxa de deformação devido à desarticulação da rede de polímero e ao alinhamento parcial das macromoléculas na direção do fluxo cisalhante.

\subsubsection{Parâmetros reológicos}

$\mathrm{Na}$ tabela 5 foram reunidas as propriedades reológicas dos dois fluidos. Analisando esta tabela, é possível observar que o fluido FGX apresentou maiores viscosidades, limite de escoamento e força gel, o que está em concordância com o resultado apresentado na Tabela 2, em que o fluido FGX apresentou maior índice de consistência, que faz com que a viscosidade aparente do fluido aumente e, consequentemente, sua resistência ao escoamento. Ou seja, por ser mais 
viscoso, ele possui maior capacidade de suspensão dos cascalhos e requer uma maior força para escoar.

Tabela 5: Propriedades reológicas dos fluidos FGX e FBT.

\begin{tabular}{|c|c|c|c|c|c|}
\hline Fluido & $\mathrm{VA}(\mathrm{cP})$ & VP (cP) & LE (lbf/100ft $\left.{ }^{2}\right)$ & $\mathrm{G}_{0}\left(\mathrm{lbf} / 100 \mathrm{ft}^{2}\right)$ & $G_{F}\left(l b f / 100 f^{2}\right)$ \\
\hline FGX & 133,13 & 109,25 & 47,75 & 8 & 10,5 \\
\hline FBT & 69,63 & 63,25 & 12,75 & 4,25 & 7 \\
\hline
\end{tabular}

Apesar de não existir valores ideais de viscosidades e limite de escoamento, já que este controle depende da operação, pode-se considerar que o fluido FGX, que foi viscosificado com goma xantana, apresentou melhores resultados, uma vez que fez uso de menor porcentagem de viscosificante, apenas 1,5\%, e apresentou maiores viscosidades e força gel, além de apresentar maior interação com o sistema água/glicerina.

\section{CONCLUSÃO}

Os fluidos de perfuração microemulsionados FGX e FBT, aditivados com goma xantana e bentonita, respectivamente, apresentaram comportamento pseudoplástico e o modelo de Herschel-Bulkley foi o que melhor se ajustou ao comportamento dos fluidos, caracterizando-os como fluidos de potência com limite de escoamento inicial.

A bentonita não apresentou interação com a glicerina da solução aquosa de glicerina do sistema. A goma xantana mostrou que aumenta a viscosidade com o aumento da quantidade adicionada à solução e que as viscosidades aparente e plástica do sistema crescem nas primeiras $24 \mathrm{~h}$, decrescem nas $24 \mathrm{~h}$ seguintes e atingem a estabilidade em seguida.

O fluido aditivado com goma xantana mostrou maior pseudoplasticidade, viscosidade, limite de escoamento e força gel, com uma menor quantidade do aditivo, indicando ser mais eficaz na viscosificação do sistema microemulsionado.

\section{REFERÊNCIAS}

Amorim, L. V. (2003). Melhoria, Proteção e Recuperação da Reologia de Fluidos Hidroargilosos para Uso na Perfuração de Poços de Petróleo. (Tese de Doutorado). Universidade Federal de Campina Grande, UFCG. Campina Grande, PB, Brasil.

Araújo, T. A., Faria, S., França, F. P., Cardoso, V. L. (2005). Avaliação do comportamento reológico dos diferentes meios de produção de goma xantana a partir do caldo de cana. Congresso Brasileiro de Engenharia Química em Iniciação Científica, Campinas, SP, Brasil, 6. 
Assis, D. J. (2014). Influência da aeração e agitação nas propriedades de gomas xantanas produzidas por Xanthomonas campestres mangiferaeindicae $2103 \mathrm{com}$ glicerina residual do biodiesel: otimização e cinética do bioprocesso. (Dissertação de mestrado). Universidade Federal da Bahia, UFBA. Salvador, BA, Brasil.

Borges, C. D., Vendruscolo, C. T. (2009). Comportamento Reológico de Xantana Produzida por Xanthomonas arboricola pv pruni para Aplicação em Fluido de Perfuração de Poços de Petróleo. Polímeros: Ciência e Tecnologia. 19 (2), 160 - 165.

Choo, K. Y., Bai, K. (2015). Effects of bentonite concentration and solution pH on the rheological properties and long-term stabilities of bentonite suspensions. Applied Clay Science. 108, 182 $-190$.

Corrêa, C. C., Cruz, G. F., Vaz Júnior, A. S. L., Araújo, B. S. A., Silva, A. A., Rodrigues, R. A., Lomba, R. F. T., Waldmann, A. T. A. (2017). Avaliação do potencial uso de bioglicerina como base para formulação de fluidos de perfuração aquosos para poços de petróleo e gás. Química Nova, 40 (4), $378-387$.

Cremasco, M. A. (2012). Operações unitárias em sistemas particulados e fluidomecânicos. São Paulo: Blucher.

Cruz, G. F., Corrêa C. C., Vaz Júnior, A. S. L., Lomba, R. F. T., Vianna, A. M. (2015, novembro). Fluido de perfuração aquoso base glicerina para a construção de poços de petróleo e gás. BR102015030667-9. INPI.

Curbelo, F. D. S., Garnica, A. I. C., Freitas, J. C. O., Sousa, R. P. F., Braga, G. S. (2016, agosto). Desenvolvimento e características de um fluido de perfuração microemulsionado a base de glicerina. BR1020160190487. INPI.

Daltin D. (2011). Tensoativos: Química, propriedades e aplicações. São Paulo: Blucher.

Davies S. N., Meeten G. H., Way P. W. (1998, julho). Water based drillimg fluid additive and methods of using fluids containing additives. 5652200. UNITED STATES PATENT.

García-Ochoa, F., Santos, V. E., Casas, J. A., Gomes, E. (2000). Xanthan gum: production, recovery and properties. Biotechnology Advanced, 18, 549-579.

Hamed S. B., Belhadri, M. (2009). Rheological properties of biopolymers drilling fluids. Journal of Petroleum, Science and Engineering, 67, 84-90.

Hayes J. B., Haws G. W., Gogarty W. B. (1977, março). Water-in-oil microemulsion drilling fluids. 4012329. UNITED STATES PATENT.

Kelessidis, V. C., Maglione, R., Tsamantaki, C., Aspirtakis, Y. (2006). Optimal determination of rheological parameters for Herschel-Bulkley drilling fluids and impact on pressure drop, velocity profiles and penetration rates during drilling. Journal of Petroleum Science and Engineering, 53, $203-224$.

Ki-Won, S., Yong-Seok, K., Gap-Shik, C. (2006). Rheology of concentrated xanthan gum solutions: steady shear flow behavior. Fibers and Polymers, 7 (2), 129-138. 
Machado, J. C. (2002). Fundamentos e Classificação de Fluidos Viscosos. Reologia e Escoamento de Fluidos - Ênfase na indústria do petróleo. Rio de Janeiro: Interciência.

Melo, K. C., Dantas, T. N. C., Barros Neto, E. L. (2013). Influência da temperatura na reologia de fluidos de perfuração preparados com carboximetilcelulose, goma xantana e bentonita. Holos, 5.

Mittal K. L. (1979). Solution Chemistry of Surfactants. New York: Plenum Press.

Nascimento, R. C. A. M., Amorim, L. V., Santana, L. N. L. (2010). Desenvolvimento de fluidos aquosos com bentonita para perfuração de poços de petróleo onshore. Cerâmica, 56, 179 187.

Norma API 13 B. (2012). Recommended Practice for Field Testing Oil-Based Drilling Fluids. API Recommended Practice 13B-2. 5 ed.ROSS, C. S., SHANNON, E. V. (1926). Minerals of Bentonite and Related Clays and Their Physical Properties. Journal of American Ceramic Society 9, 77.

Oliveira, M. A. M., Mothé, C. G., Oliveira, L. G. (2015). Petroleum Patent Used Such Monitoring Technological Like Innovation Process of Drilling Fluids with Xanthan Gum - World Panorama. Engineering, 7, 395 - 407.

Santos, P. S. (1989). Ciência e Tecnologia de Argilas. São Paulo: Blucher.

Silva, A. R. V., Ferreira, H. S. (2008). Argilas bentoníticas: conceitos, estruturas, propriedades, usos industriais, reservas, produção e produtores/fornecedores nacionais e internacionais. Revista Eletrônica de Materiais e Processos, 3 (2), 26 - 35.

Silva, C. T. (2003). Desenvolvimento de fluidos de perfuração a base de óleos vegetais (Trabalho final de curso de Engenharia Química). Universidade Federal do Rio Grande do Norte, UFRN, Natal, RN, Brasil.

Souza, G. S, Luporini, S., Rigoli, I. C. (2017). Caracterização reológica de suspensões argilosas salinas com goma xantana para fluidos de perfuração de poços de petróleo. Revista Matéria, 22 (1).

Young, R. J. (1981). Introduction to polymer. New York: Chapman \& Hall. 